\title{
SENSOR SYSTEM TO MONITOR AMMONIA
}

Final Technical Report

John G. Edwards

PHOTONIC SENSOR SYSTEMS, INC.

573-B Courtland Street, NE

Atlanta, Georgia 30308

Date Published — October 1999

PREPARED FOR THE UNITED STATES

DEPARTMENT OF ENERGY

Under Contract No. DE-FC36-94GO10005 


\section{DISCLAIMER}

This report was prepared as an account of work sponsored by an agency of the United States Government. Neither the United States Government nor any agency thereof, nor any of their employees, make any warranty, express or implied, or assumes any legal liability or responsibility for the accuracy, completeness, or usefuiness of any information, apparatus, product, or process disclosed, or represents that its use would not infringe privately owned rights. Reference herein to any specific commercial product, process, or service by trade name, trademark, manufacturer, or otherwise does not necessarily constitute or imply its endorsement, recommendation, or favoring by the United States Government or any agency thereof. The views and opinions of authors expressed herein do not necessarily state or reflect those of the United States Government or any agency thereof. 


\section{DISCLAIMER}

Portions of this document may be illegible in electronic image products. Images are produced from the best available original document. 


\section{TABLE OF CONTENTS}

List of Figures................................................................................. ii

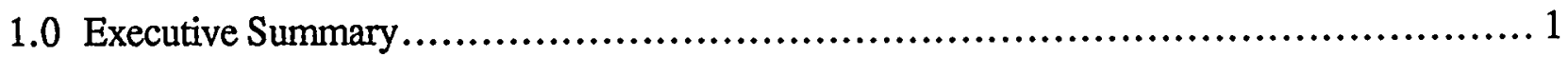

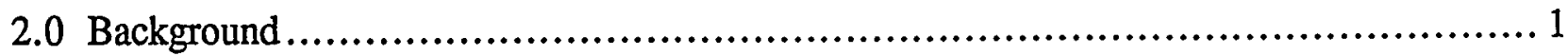

2.1 Sensor Concept ....................................................................... 1

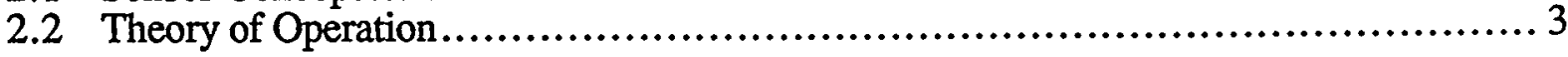

3.0 Description of Prototype Ammonia Sensor ............................................. 5

3.1 Overview ............................................................................. 5

3.2 Optics Subassembly ................................................................. 6

3.3 Electronics Subassembly ................................................... 8

4.0 Sensing Chemistry and Experimental Results ........................................ 9

4.1 Overview .......................................................................... 9

4.2 Polyethyleneimine/Citric Acid Sensing Chemistry ................................. 10

4.3 Poly(4-vinyl phenol) Sensing Chemistry ........................................ 12

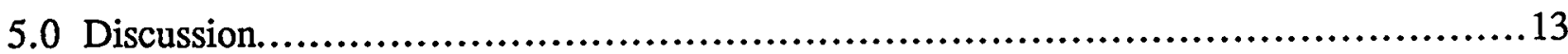

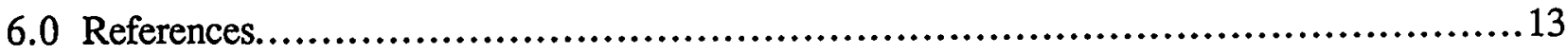




\section{LIST OF FIGURES}

Figure 1. Cross-section of an IO waveguide............................................ 2

Figure 2. Hartman interferometer configuration......................................... 2

Figure 3. Cross-section of an IO interferometric sensor package .............................. 3

Figure 4. Response to surface index changes as a function of waveguide thickness .............. 4

Figure 5. Prototype ammonia sensor package .......................................... 5

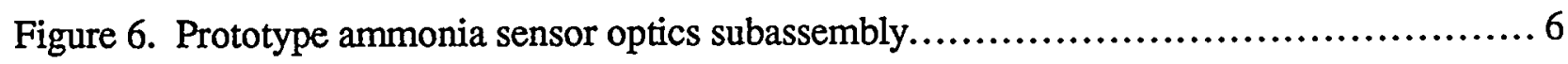

Figure 7. 13-channel integrated optic sensing chip......................................

Figure 8. Hartman interferometer detail structure (not to scale) ............................ 8

Figure 9. Prototype ammonia sensor electronics subassembly ............................... 9

Figure 10. Polyethyleneimine/citric acid sensing chemistry............................... 10

Figure 11. Response of polyethyleneimine/citric acid to various ammonia concentrations.........11

Figure 12. Ammonia calibration curve for polyethyleneimine/citric acid chemistry...............11

Figure 13. Response of poly(4-vinyl phenol) to ammonia at $60 \mathrm{ppmv}$.......................12

Figure 14. Ammonia calibration curve for poly(4-vinyl phenol) chemistry .....................12 


\subsection{EXECUTIVE SUMMARY}

This final technical report summaries the results of a project to develop a prototype integrated optic ammonia $\left(\mathrm{NH}_{3}\right)$ sensor for agricultural and industrial applications. The majority of the support for this project was provided by the Department of Energy (DOE) through Cooperative Agreement \# DE-FC36-94GO10005. The sensor is based on a planar integrated optic waveguide interferometer developed at the Georgia Tech Research Institute (GTRI). The feasibility of using this technology for detecting ammonia was first demonstrated at GTRI with assistance from DOE through Cooperative Agreement \# DE-FC02-89ID12905. This project is a continuation of that work.

The overall goal is to develop a gas-phase ammonia sensor suitable for: 1) monitoring gaseous nitrogen transfer from agricultural croplands and 2) facilitating the use of ammonia in a variety of industrial applications such as ammonia production and large scale refrigeration. The specific objective of this phase of the project was to fabricate a prototype ammonia sensor and evaluate its accuracy and reliability in real-world settings. Four major tasks were planned: design and fabrication, testing and characterization, evaluation, and reporting.

A highly sensitive prototype integrated optic (IO) sensor for detection of gaseous ammonia has been developed and demonstrated. The Hartman interferometer [1,2] on which the sensor is based directly detects refractive index changes of less than $10^{-6}$ occurring on or at its surface. The combination of the inherent stability of the integrated optic platform, the outstanding sensitivity of the interferometer and incorporation of active surface chemistries on the waveguide surface results in chemical detection capabilities in the low part-per-billion by volume ( $p p b v)$ range for ammonia. Prototype sensors were produced based on a 13-channel IO chip. Water vapor was found to be a significant interferant. To counter the water vapor interference, reference channel chemistry was developed for optical subtraction of the interfering water signal from the ammonia measurements. Device sensitivity was found to be somewhat dependent on the ambient relative humidity, but in general, the prototype sensor is capable of sensing gaseous ammonia at concentrations of less than $100 \mathrm{ppbv}$. Although some further optimization is required, the planar configuration provides a format that is easily manufactured, and most importantly, lends itself to practical applications. Unfortunately, the work needed to address humidity interference consumed all of the resources available for this phase of the project, and it was not possible to conduct the planned agricultural and industrial evaluations.

The detailed description of the prototype IO interferometer sensor system, and the sensing chemistry and experimental results are detailed in the following sections of this report.

\subsection{BACKGROUND}

\subsection{Sensor Concept}

The IO interferometric transducer relies on detection of a refractive index change at or on the surface of a planar optical waveguide. These devices fall into the so-called evanescent field sensing technologies wherein an electric field penetrates into the superstrate above the waveguide and serves as a probe beam. For chemical sensing applications, the waveguide surface is typically functionalized with a reactive chemistry designed to react reversibly with an analyte of interest. The reversible reaction has associated with it a refractive index change to which the evanescent field is quite sensitive.

The basic waveguide interaction and configuration is illustrated in Fig. 1. Due to total internal reflection at the dielectric interfaces, light coupled into the waveguide layer is confined and guided. The coupled light possesses an evanescent field distribution that decays exponentially into both the substrate and cover layers. Interaction of a target analyte with the chemically selective coating on 
the chip surface produces a phase change in this guided optical wave. Performance of the IO sensor is critically dependent on the optical waveguide properties. The degree to which the target analyte affects the phase of the guided optical wave is a function of both the refractive index difference between the waveguide and substrate layers and the thickness of the waveguide layer. In general, optimal sensitivity is achieved using a large index difference between the waveguide and substrate layers, and by making the substrate index less than that of the chemically selective coating.

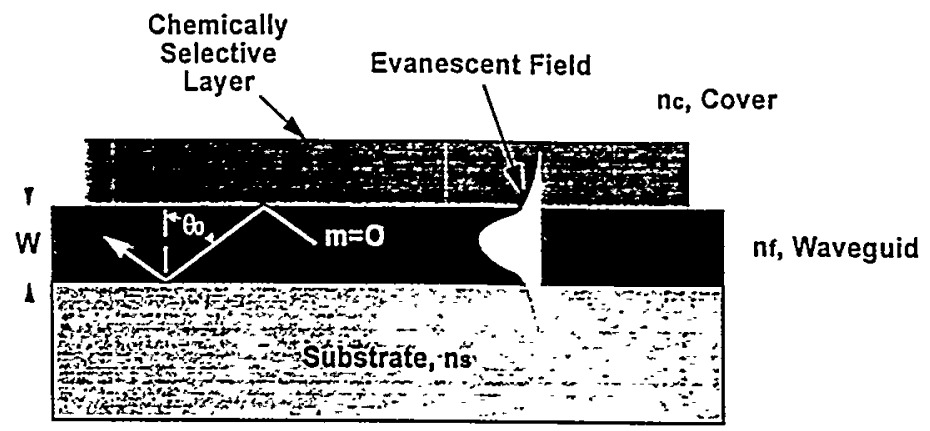

Figure 1. Cross-section of an IO waveguide.

The phase delay introduced to the guided wave as a result of interaction with a target analyte is easily detected by means of an optical circuit known as an interferometer. The Hartman interferometer configuration used for the prototype ammonia sensor is illustrated in Fig. 2. Key elements include grating couplers, the waveguide film and beam splitters/beam combiners. The optical input is coupled into the waveguide by the couplers and the propagating optical wave traverses the length of the device under the signal and reference channels defined on the surface of the waveguide. The beam splitting/beam combining elements divide and mix the reference and signal waves to generate the interference. Any phase delays due to interactions of an analyte with the surface chemistry on the signal arm is easily observed through the resulting changes in the interference pattern.

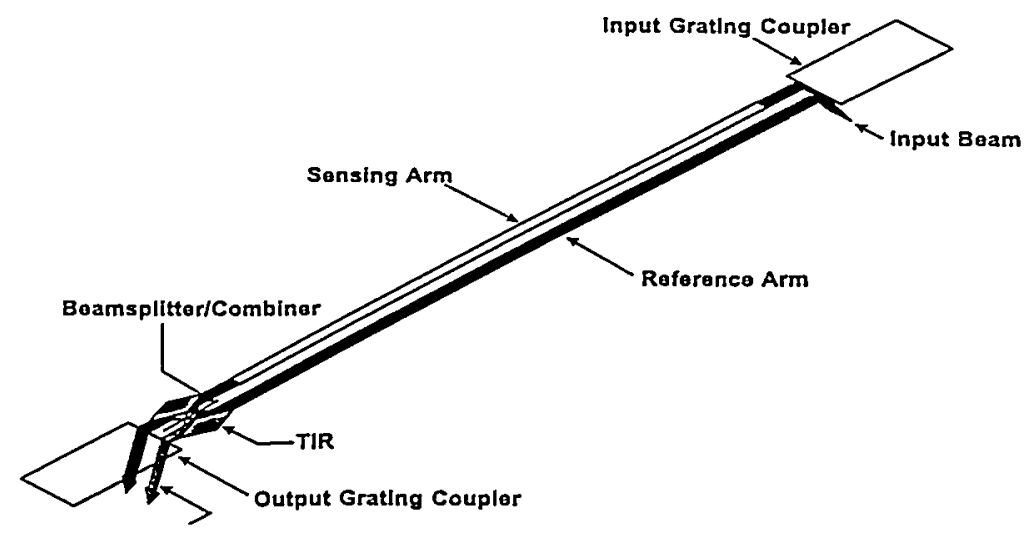

Figure 2. Hartman interferometer configuration. 
A basic IO sensor package based on this configuration is illustrated conceptually in Fig. 3. The major components include an IO chip, a diode laser and a photodiode detector. The only component not shown, but essential to a complete sensor system, is the signal processing electronics. In a typical sensor configuration, the waveguide is a thin film $(<200 \mathrm{~nm})$ prepared from an optically transparent, high refractive index material (such as silicon nitride) that is deposited on a planar optical glass substrate. The chemically selective coating is deposited on the surface of the waveguide may vary in thickness from one monolayer of molecules to more than $500 \mathrm{~nm}$. Grating couplers are utilized to couple the diode laser output into and out of the waveguide. The grating couplers are essential to ensuring a practical field instrument. Because the waveguide sensing films tend to be extremely thin, the traditional end-fire or butt-coupling methods require precision XYZ alignment. Conversely, the grating coupler method requires only a relatively coarse angular alignment and is sufficiently simple such that field replacement of an IO chip becomes possible.

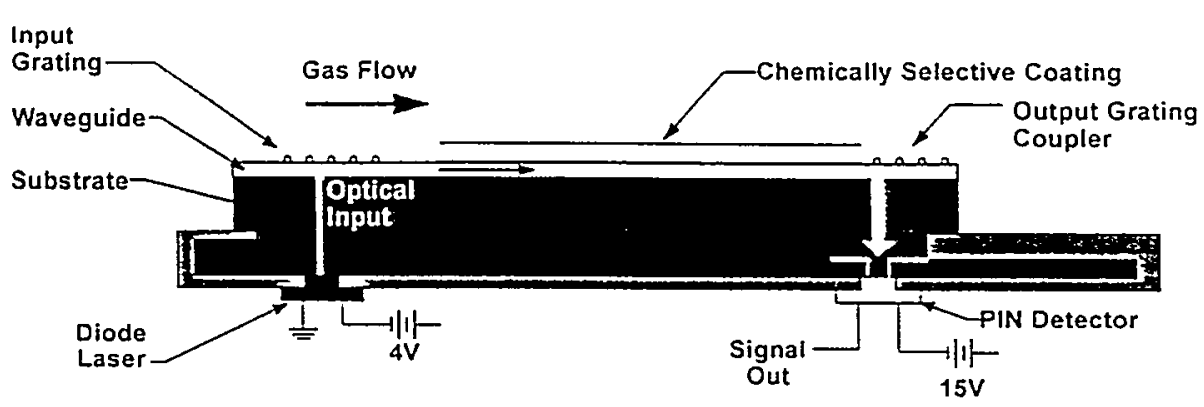

Figure 3. Cross-section of an IO interferometric sensor package.

\subsection{Theory of Operation}

Operation of the IO sensor can be described quantitatively using a dispersion relationship that incorporates the step index waveguide parameters $n_{f}$ (waveguide film index), $n_{c}$ (cover film index), $\mathrm{n}_{\mathrm{s}}$ (substrate index) and $\mathrm{W}$ (waveguide film thickness) [3]. The dispersion relationship can be derived from an electromagnetic boundary value problem where the guided wave is confined by two dielectric boundaries. It is defined by the "transverse resonance condition", which requires the sum of all phase shifts perpendicular to the direction of propagation in the waveguide to be a multiple of $2 \pi(2 \pi \mathrm{m}$, where $\mathrm{m}=0,1,2, \ldots)$ for one "zig-zag" period of the light propagating within the waveguide. For each full period (two transverse passages), a phase shift of $2 \mathrm{kn}_{\mathrm{f}} \mathrm{W} \cos \theta$ occurs. Additionally, phase shifts of $-2 \theta_{c}$ and $-2 \theta_{s}$ occur due to total internal reflection at the cover and substrate boundaries of the waveguide, as shown above. Thus:

$$
\begin{aligned}
& 2 \mathrm{kn}_{\mathrm{f}} \mathrm{W} \cos \theta-2 \theta_{\mathrm{c}}-2 \theta_{\mathrm{S}}=2 \pi \mathrm{m} \\
& \text { where } \quad \theta_{\mathrm{c}}=\tan ^{-1}\left[\left(\mathrm{n}_{\mathrm{f}} 2 \sin ^{2} \theta-\mathrm{n}_{\mathrm{c}}{ }^{2}\right)^{1 / 2} / \mathrm{n}_{\mathrm{f}} \cos \theta\right] \\
& \text { and } \quad \theta_{\mathrm{S}}=\tan ^{-1}\left[\left(\mathrm{n}_{\mathrm{f}}{ }^{2} \sin ^{2} \theta-\mathrm{n}_{\mathrm{s}}{ }^{2}\right)^{1 / 2} / \mathrm{n}_{\mathrm{f}} \cos \theta\right]
\end{aligned}
$$

Because the waveguide is thin and the index differences are small, waveguiding occurs only at discrete values of $\theta$ with each discrete angle corresponding to a propagating mode. The term $n_{f} \sin \theta$ is defined as the "effective mode index" $\left(\mathrm{N}_{\text {eff }}\right)$ and represents an effective refractive index the waveguide material system and the cover film exhibits for a propagating mode of a specific optical 
wavelength. If $n_{f}, n_{s}$, and $W$ are held constant while $n_{c}$ is varied (i.e., a refractive index change in the cover medium), then only $\theta$ can be varied to satisfy the conditions for propagation of the light ray through the waveguide. This change in the cover index manifests itself as a change in $\mathrm{N}_{\text {eff }}$, which is easily detected using interferometric techniques.

In practice, the minimal detectable change in $\mathrm{N}_{\text {eff }}$ is defined by the minimum phase shift detectable by the interferometer. The output of a waveguide interferometer operating at quadrature is described by the following equation:

$$
\mathrm{I}=\left(\mathrm{I}_{0} / 2\right)\left\{1+\cos \left[(\pi / 2)+(2 \pi / \lambda) \mathrm{L}\left(\Delta \mathrm{N}_{\mathrm{eff}}\right)\right]\right\}
$$

where $I$ is the output intensity of the interferometer, $I_{0}$ is the peak output intensity, $\lambda$ the free space wavelength of guided light, $\mathrm{L}$ is the interferometer path length, and $\Delta \mathrm{N}_{\text {eff }}$ represents the difference in the effective waveguide index induced by the surface index change due to the analyte interaction.

Using the previous equations, integrated optic interferometers based on specific material systems can be designed with enhanced sensitivity to surface index changes. In general, high sensitivity requires a thin high index waveguide film on a low index substrate. The objective is to maximize the overlap of the evanescent field with the chemically selective surface layer. Sensitivity as a function of waveguide film thickness is presented in Fig. 4 for a silicon nitride $\left(\mathrm{Si}_{3} \mathrm{~N}_{4}\right)$ waveguide and fused silica substrate. The calculations were based on the binding of a $5 \mathrm{~nm}$ sensing film ( $\mathrm{n}=1.48)$ attached to the waveguide surface. The waveguide film index was assumed to have a refractive index of 1.90 and the substrate to have a refractive index of 1.457 . The results indicate a waveguide film thickness of approximately $140 \mathrm{~nm}$ offers maximum sensitivity. Detection sensitivity could be further enhanced by the use of a higher index waveguide film such as tantalum pentoxide. However, scattering effects due to the stronger interaction of the evanescent field with the surface chemistry will eventually limit utility.

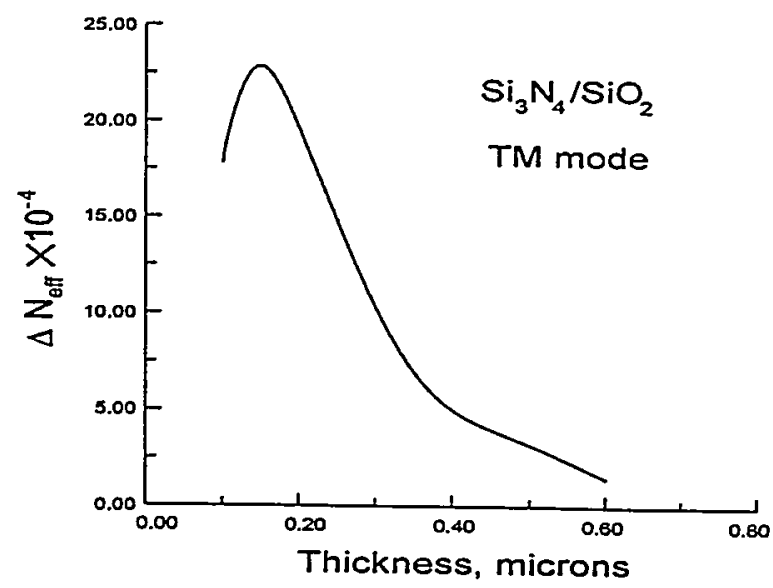

Figure 4. Response to surface index changes as a function of waveguide thickness. 


\subsection{DESCRIPTION OF PROTOTYPE AMMONIA SENSOR}

\subsection{Overview}

The fully packaged prototype ammonia sensor system is shown in Fig. 5. The package comprises three major components: an optics subassembly, and electronics subassembly and a durable housing. The overall dimensions are $18.5 \mathrm{~cm} \times 15 \mathrm{~cm} \mathrm{x} 7 \mathrm{~cm}$. The package has an RS232 port for interface to a standard "Wintel" personal computer, which is used solely for system setup and data display. An external $5 \mathrm{~V}$ power supply is required.

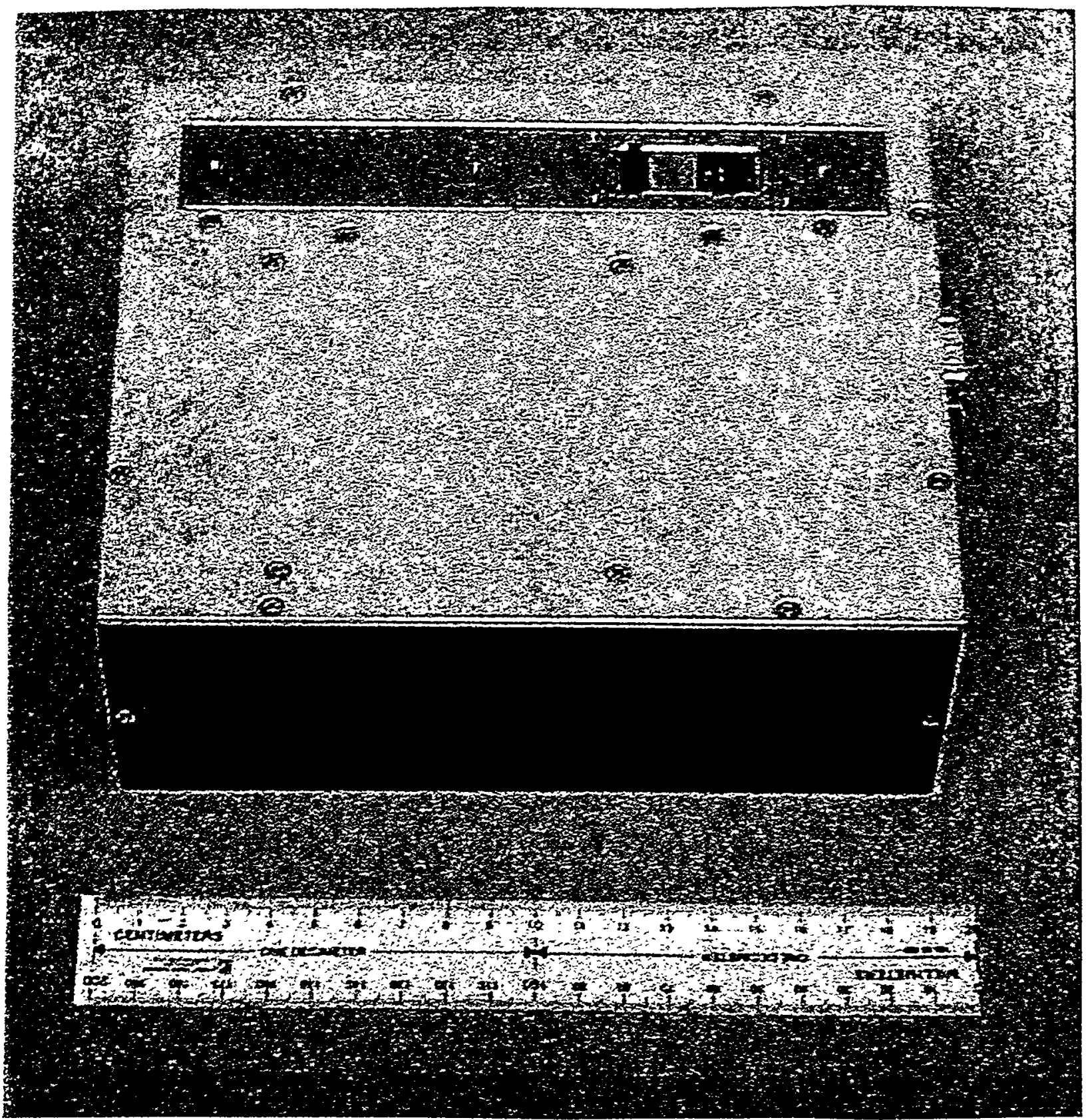

Figure 5. Prototype ammonia sensor package. 


\subsection{Optics Subassembly}

A picture and a schematic illustration of the prototype ammonia sensor optics subassembly are shown in Fig. 6. This subassembly includes a 13-channel integrated optic sensing chip, an Hitachi $670 \mathrm{~nm}, 10 \mathrm{~mW}$ diode laser and a Texas instruments TSL401 128-element photodiode array, all housed in a rigid, stainless steel mechanical structure. The optics subassembly dimensions are $16.5 \mathrm{~cm} \times 2.5 \mathrm{~cm} \times 2.0 \mathrm{~cm}$.
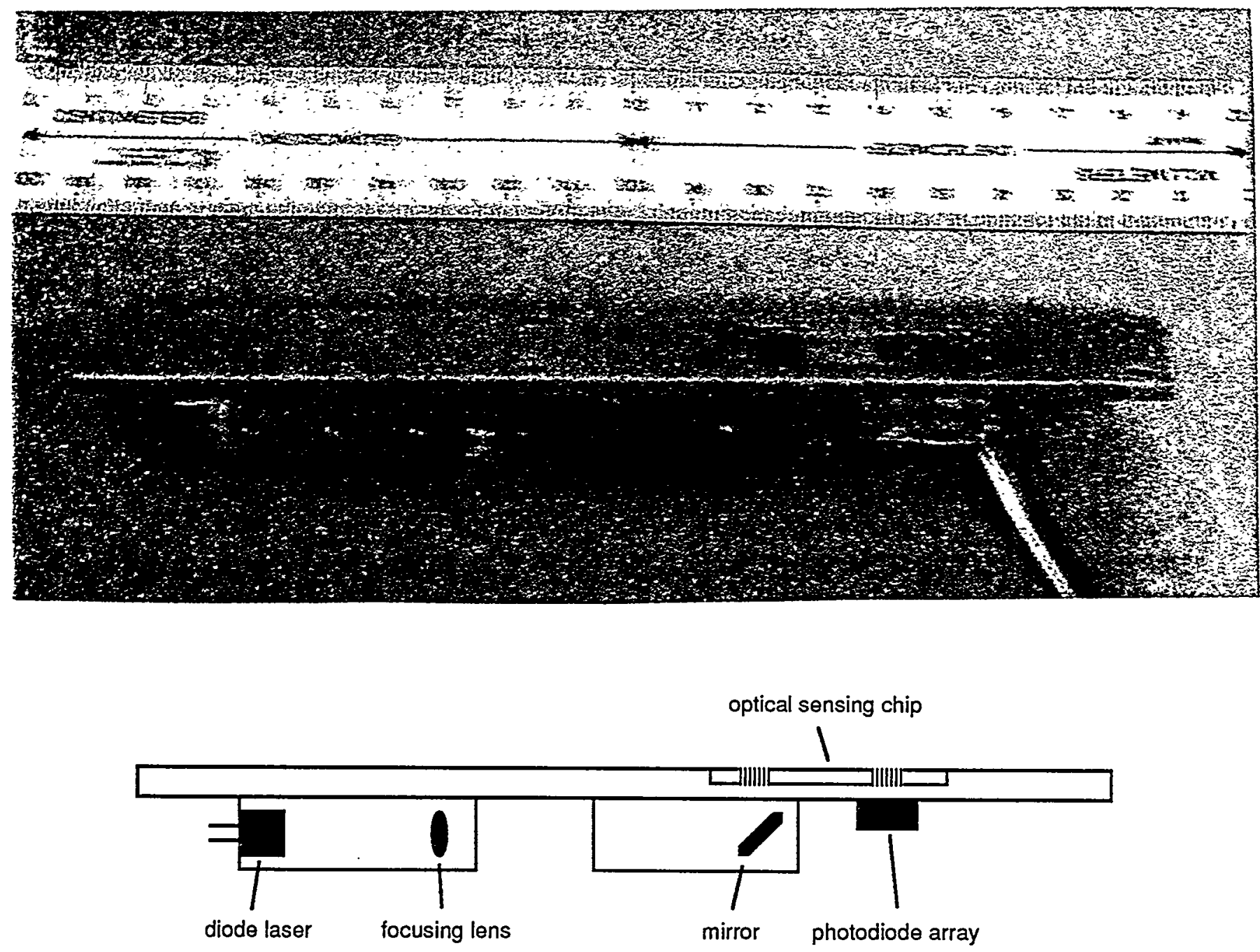

Figure 6. Prototype ammonia sensor optics subassembly.

The key element of the optics subassembly is the 13-channel integrated optic sensing chip. A picture and a schematic illustration of the optical chip are shown in Fig. 7. The chip contains 13 separate Hartman interferometers. Twelve of the interferometers (labeled 1 through 12) are coated with chemically selective films. The availability of multiple interferometers allows for redundant ammonia sensing channels to improve signal processing and/or nulling channels to cancel known interferants. The thirteenth interferometer (labeled 0 ) is completely isolated from the environment by a thick overcoating $(500 \mathrm{~nm})$ of silicon dioxide. It is used to compensate for non-chemical effects such as fluctuations in the diode laser wavelength or large temperature excursions. (The design of the Hartman interferometer is inherently insensitive to small temperature excursions.) 
The signal and reference arms of each interferometer are $190 \mu \mathrm{m}$ wide, with $190 \mu \mathrm{m}$ gaps between the arms, and they vary in length from $0.01 \mathrm{~cm}$ to $1 \mathrm{~cm}$, depending on the desired sensitivity. The overall dimensions of the optical sensing chip are $3.5 \mathrm{~cm}$ long by $1.5 \mathrm{~cm}$ wide.


Figure 7. 13-channel integrated optic sensing chip. 
As illustrated in Fig. 7, up to three interferometers can be devoted each selective chemistry to ensure adequate sensitivity and adequate dynamic range. The three interferometers are referred to as coarse, medium and fine. The coarse interferometer is the shortest, giving a proportionately small response. The fine interferometer is the longest, giving a proportionately large response. The output from the coarse interferometer provides an unambiguous (but low-resolution) measure of the analyte concentration across the entire dynamic range. The outputs from the medium and fine interferometers provide successively improved resolution. The sensor electronics process and combine the three outputs to give a high-resolution response across the entire dynamic range (see below).

The detailed structure of a single Hartman interferometer is shown in Fig. 8. It consists of grating couplers and beam combining/beam splitting elements. The grating couplers are critical to achieving optimal coupling of light into and out of the optical chip. The beam splitting/combining elements divide and mix the reference and signal waves to generate the interference. The input/output couplers are produced by reactive ion etching (RIE) a $700 \mathrm{~nm}$ period grating into the substrate surface. The waveguiding film is then deposited on the substrate using a plasma enhanced chemical vapor deposition process (PECVD). A $140 \mathrm{~nm} \mathrm{Si}{ }_{3} \mathrm{~N}_{4}$ film is typically used for the waveguides. The beam splitting/beam combing elements consist of total internal reflecting (TIR) mirrors and a beam splitter (BS). The TIR is fabricated by etching a $40 \mu \mathrm{m}$ wide trough completely through the $\mathrm{Si}_{3} \mathrm{~N}_{4}$ film. The beam splitter is generated by etching a $4 \mu \mathrm{m}$ wide trough approximately $50 \mathrm{~nm}$ into the waveguide film. The entire surface area of the optical chip, with the exception of the sensing regions, is then overcoated with a $500 \mathrm{~nm}$ thick silicon dioxide $\left(\mathrm{SiO}_{2}\right)$ film. The $\mathrm{SiO}_{2}$ isolates the grating couplers, TIR mirrors and beam splitters to ensure their performance is insensitive to environmentally induced changes at or on the waveguide surface. The performance of the TIR mirrors is critically dependent on the existence of smooth walls vertical with respect to the substrate surface to within \pm 1 arc degree.

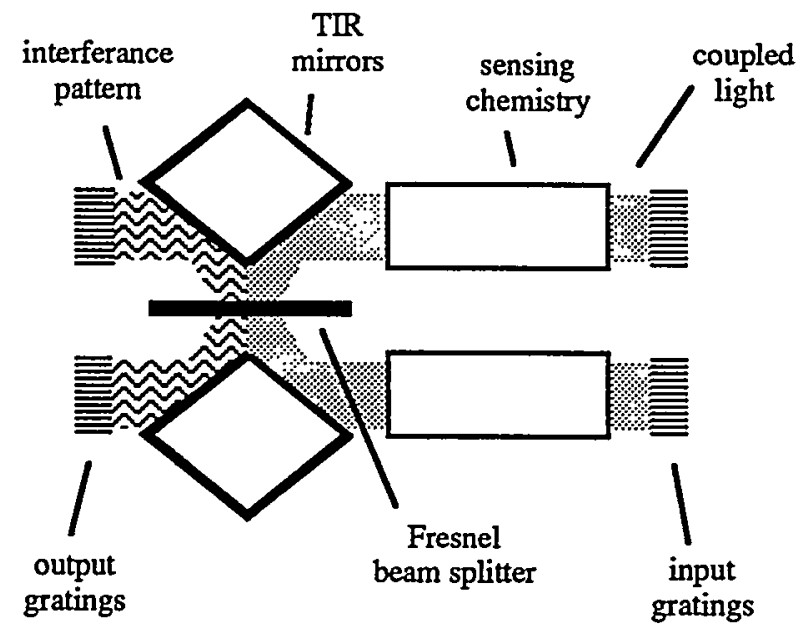

Figure 8. Hartman interferometer detail structure (not to scale).

\subsection{Electronics Subassembly}

A picture of the prototype ammonia sensor electronics subassembly is shown in Fig. 9. The electronics are a two-board set. The lower board is an Advanced Micro. Devices PC-104 standard board computer (66 MHz CPU; 1 Mbyte RAM). The upper board is custom built, providing a driver and active control for the diode laser, a clock and control signals for all 128 elements of the TSL1401 photodiode array, and signal processing capabilities. The electronics subassembly dimensions are $9.5 \mathrm{~cm} \times 9 \mathrm{~cm} \times 2.0 \mathrm{~cm}$. 


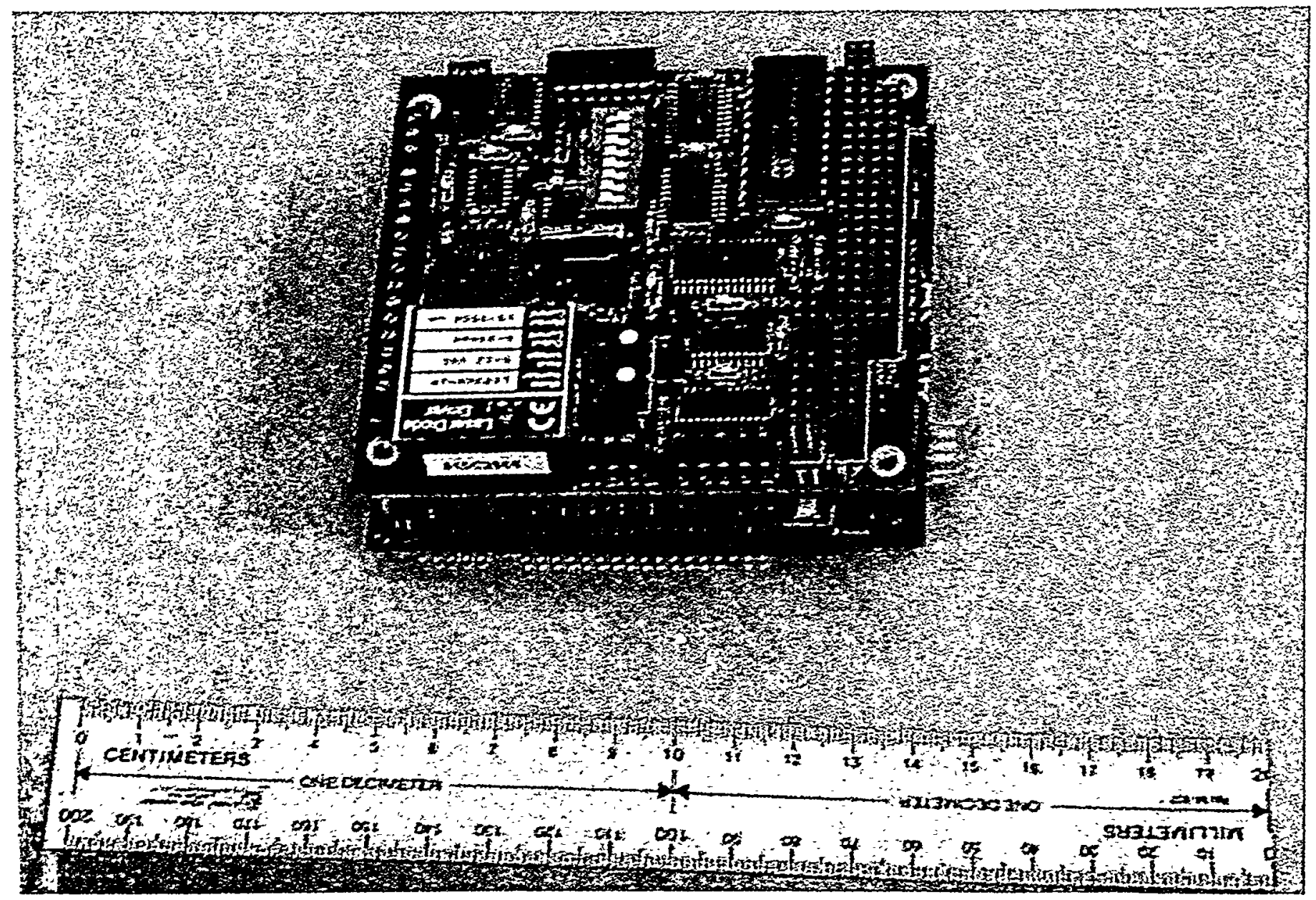

Figure 9. Prototype ammonia sensor electronics subassembly.

The electronics implement a three-step signal processing algorithm. First, the raw optical intensities from each of the thirteen interferometers on the integrated optic sensing chip are captured and digitized. Second, the digitized intensities are deconvolved into the underlying phase shift associated with each set of coarse, medium and fine channels (corrected for the phase shift in the buried reference interferometer). Third, a pre-determined calibration curve is used to translate the phase shifts into an ammonia concentration in meaningful engineering units (i.e.; ppbv). The electronics subassembly is capable outputting concentrations at a $1 \mathrm{~Hz}$ rate. (The rate limiting component is the PC-104 board computer.)

\subsection{SENSING CHEMISTRY AND EXPERIMENTAL RESULTS}

\subsection{Overview}

The chemical selectivity of the IO sensor is dependent on the chemistry applied to the interferometer sensing arm surfaces. Typically, the sensing arm surfaces are coated or functionalized with films that vary in thickness from a monolayer to a few tenths of a micron. This feature is responsible for the fast response typically observed with the sensor as it overcomes the diffusion-limited mechanisms encountered with thick films. Tailored chemistries can be passive (e.g.; inducing swelling or dissolution in a film) or active (e.g.; containing reactive or binding sites). Passive mechanisms are used when the target analyte is relatively inert (i.e.; aromatic and chlorinated hydrocarbons). Active chemistries include tailoring the acid-base strength for $\mathrm{pH}$ or amine response, and antibody-antigen binding. 
Several vapor phase ammonia detection schemes have been developed during this project using active chemistries that do not involve a change in color or fluorescence, but rather a change in index of refraction of the sensing layer. Acid-base chemistries that have been tailored for the narrow $\mathrm{pKa}$ response of ammonia are placed either on the signal arm surface or incorporated in a polymer film over the signal arm of the interferometer. A similar chemistry or polymer film is placed on the reference arm surface, except that it is tailored to be unreactive for the $\mathrm{pKa}$ response of ammonia. In this way the reference arm can be utilized to cancel interferants, which would effect both the signal and reference arm chemistries equally. Two of the most promising mechanisms are discussed below. In both cases, the ammonia sensing chemistry uses polymers coatings with two different $\mathrm{pKa}$ 's. One arm of the interferometer is tailored to have a $\mathrm{pKa}$ which lies above that of ammonia and the other arm of the interferometer is tailored to have a pKa which lies below that of ammonia. Ammonia diffuses equally into both arms but reacts with only one to produce dipoles. The interaction of the evanescent field with the induced dipole is the source of the effective index change encountered by the guided optical wave.

\subsection{Polyethyleneimine/Citric Acid Sensing Chemistry}

In the first example, citric acid was chosen to react with ammonia. Citric acid has three protons with pKa's of 3.1, 4.8 and 6.4. The proton with the pKa of 6.4 is the most useful proton to reversibly react with ammonia. The other two are too acidic to be reversible, consequently they are intentionally tied up in a reaction with a polymer adjusted to the appropriate $\mathrm{pH}$. A solution of a basic polymer, polyethyleneimine- $80 \%$ ethoxylated, $\mathrm{pH} \mathrm{11}$, is titrated with citric acid. The polymer tethers the citric acid, holding it within the evanescent field. As shown in Fig. 10, the reference arm contains polyethyleneimine- $80 \%$ ethoxylated titrated to $\mathrm{pH} 8.0$ so that no acid protons are left on the citric acid. The sensing arm is titrated with citric acid to $\mathrm{pH} 6.0$ leaving one proton on each citric acid capable of reacting with the ammonia. Ammonia reacts reversibly with these remaining citric acid protons. The polymer films are $500 \mathrm{~nm}$ thick, burying the evanescent field and shielding out background environmental effects.
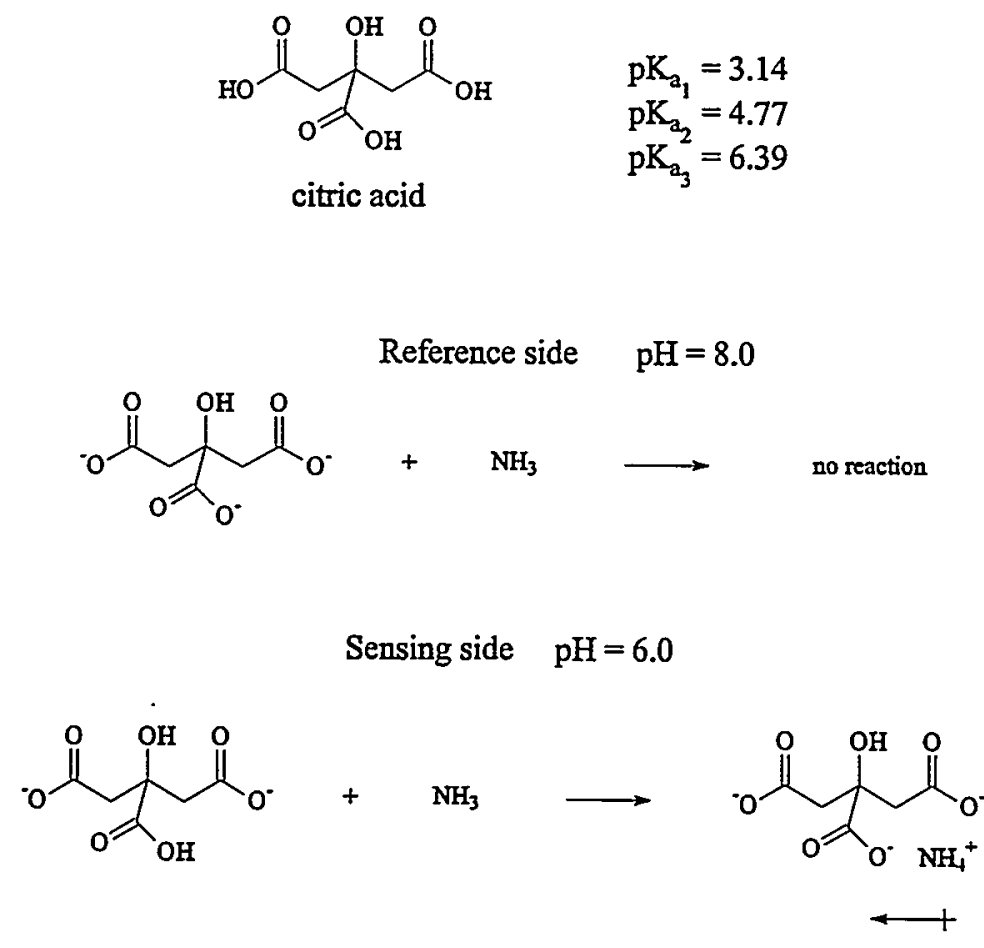

Figure 10. Polyethyleneimine/citric acid sensing chemistry. 
As shown in Figs. 11 and 12, the sensitivity of the polyethyleneimine/citric acid sensing chemistry is in the sub 100 ppbv range. This represents a substantial improvement over previously developed passive chemistries. It is theorized that water mediates the transfer of protons throughout the entire polymer matrix to the ammonia. This is born out by the fact that as relative humidity goes below $25 \%$ the sensor response greatly diminishes. The relatively slow response (compared to chemistries that have been developed of other analytes, such as $\mathrm{pH}$ ) in the useful operating range of $25-100 \%$ relative humidity is believed to stem from the less hydration of the polyethyleneimine/citric acid polymer which requires actual movement of water molecules in order to transfer the protons.

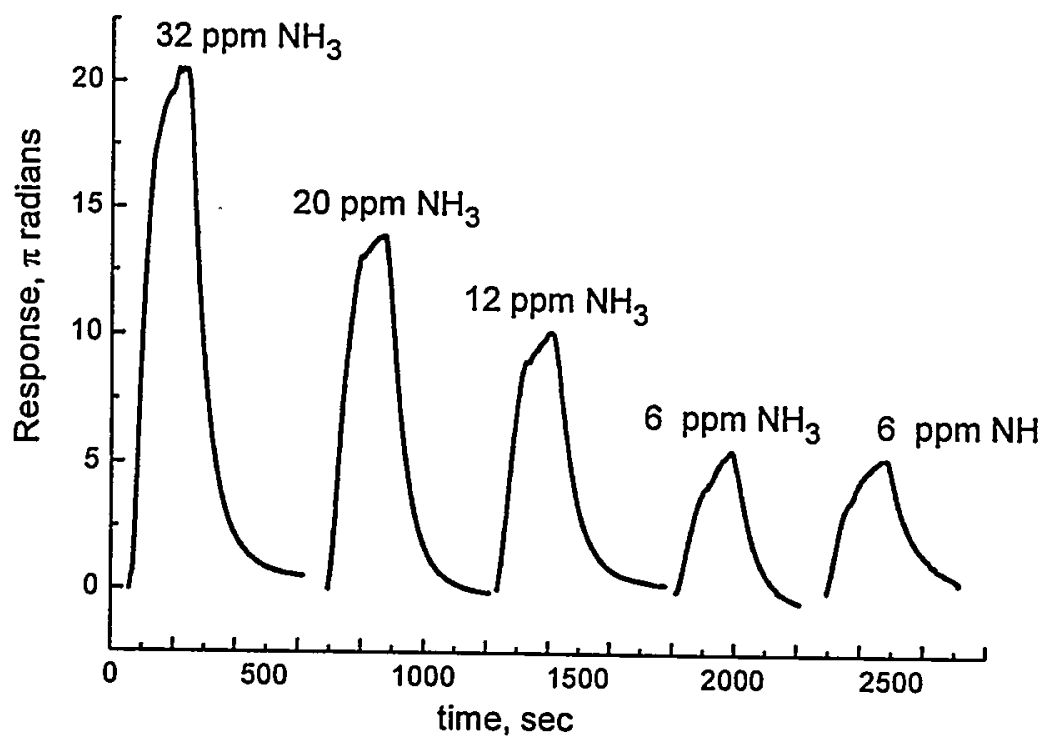

Figure 11. Response of polyethyleneimine/citric acid to various ammonia concentrations.

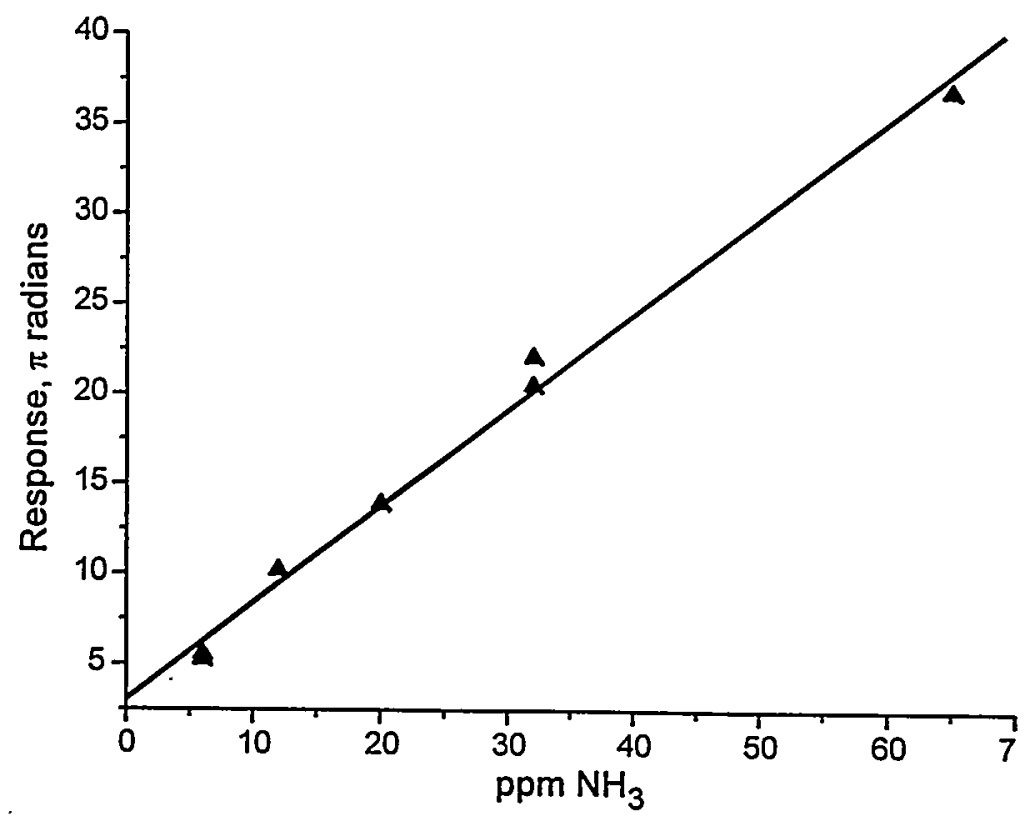

Figure 12. Ammonia calibration curve for polyethyleneimine/citric acid chemistry. 


\subsection{Poly(4-vinyl phenol) Sensing Chemistry}

To solve the response problem to ammonia at low humidity, a second approach was developed that is independent of water in the film. Poly(4-vinyl phenol) has a pKa conducive to reaction with ammonia ( $\mathrm{pKa} ~ 9.9)$. A very thin film (80 nm) of poly(4-vinyl phenol) is used to coat the sensing arm and a similarly thin film $(150 \mathrm{~nm})$ of poly (4-vinyl phenoxide, sodium salt) is used to coat the reference arm. The phenoxide has no proton to react with ammonia. Over these films is placed a $500 \mathrm{~nm}$ film of ethyl cellulose to bury the evanescent field. The ethyl cellulose allows diffusion of ammonia without participating in the reaction. As shown in Fig. 13, because the sensing film is very thin and the reaction is direct (i.e.; no water needed), the response time for the poly (4-vinyl phenol) sensing chemistry is much faster than for the polyethyleneimine/citric acid sensing chemistry. The reversal was also faster, due in part to the better matching of pKa's between ammonia and the sensing film. Further improvement could result from better tailoring of the $\mathrm{pKa}$ of the sensing film. As shown in Fig. 14, the total response of the poly(4-vinyl phenol) sensing chemistry is less than for the polyethyleneimine/citric acid sensing chemistry. This is due in part to the very thin sensing film, which doesn't take advantage of the entire evanescent field, and to smaller dipoles resulting from less separation of charge.

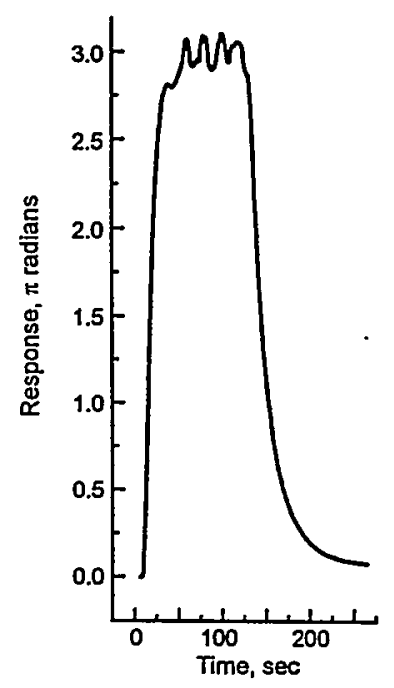

Figure 13. Response of poly(4-vinyl phenol) to ammonia at $60 \mathrm{ppmv}$.

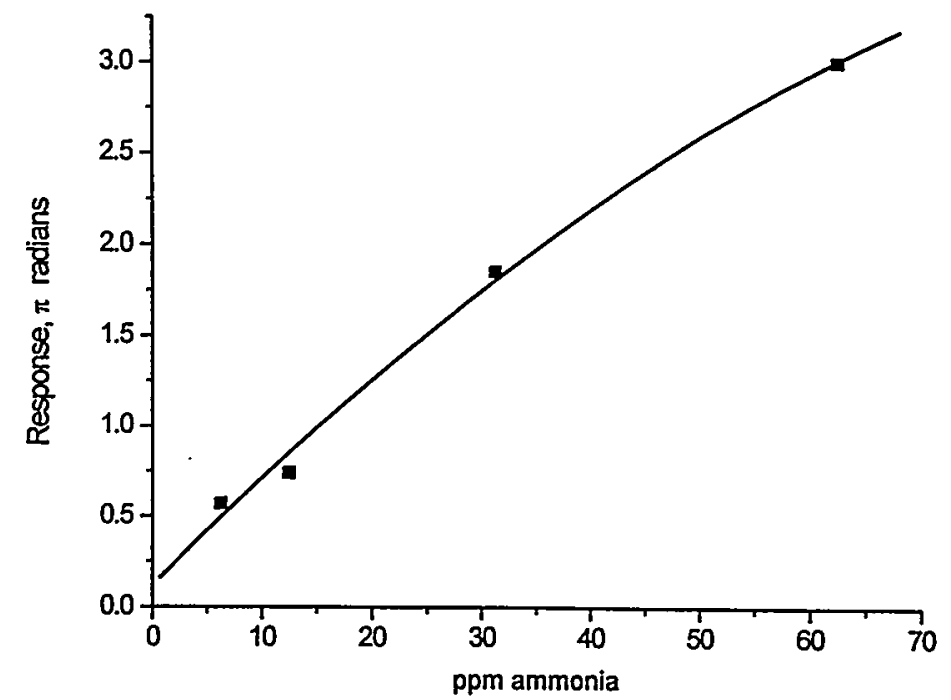

Figure 14. Ammonia calibration curve for poly(4-vinyl phenol) chemistry. 


\subsection{DISCUSSION}

The project results have shown that polymer based sensing films incorporating active acid-base sensing chemistries can provide detection sensitivities of $1 \pi$ radian phase shift for 1 to 5 ppmv of gaseous ammonia. With a base line noise level of $0.02 \pi$ radian phase shift, this translates to a lower detection limit on the order of 20 to $100 \mathrm{ppbv}$ for ammonia at a relative humidity above $25 \%$. At very low relative humidity, the response was reduce by a factor of 2 to 3 . For practically all ammonia sensing chemistries developed, water vapor was a significant interferant. It should be noted that this humidity interference is significantly different that the humidity interference experienced by conventional electrochemical ammonia sensors. There is no poisoning or consumption of the sensing moiety. A change in relatively humidity simply mimics a change in ammonia concentration. When the relative humidity returns to its original level, the humidity interference signal completely reverses, with no reduction in the IO ammonia sensor lifetime. Nevertheless, because water is so abundant and large changes in the local relative humidity can occur, the reference arm of an IO interferometric sensor was utilized to null the humidity response. Typically $90 \%$ of the humidity response could be nulled, however some humidity response always remained. The inability to completely cancel the humidity interference was largely the result of insufficient control over the signal and reference film thicknesses. In practice, with suitable polymer film deposition equipment, the coating film thicknesses should be sufficiently well controlled to cancel essentially the entire relative humidity interference.

\subsection{REFERENCES}

1 Hartman, N.F., "Optical Sensing Apparatus and Method", U.S. Patent \# 4,940,328, July 10, 1990.

2 Hartman, N.F., "Integrated Optic Interferometric Sensor", U.S. Patent \# 5,623,561, April 22, 1997.

3 Tamir, T., "Guided-Wave Optoelectronics", Springer Series in Electronics and Photonics, Volume 26, Springer-Verlag, New York. 